\title{
A Comparison of Adverse Events Among Radiofrequency Ablation, Conventional Transarterial Chemoembolization (TACE) and Drug-Eluting Bead TACE in Treating Hepatocellular Carcinoma Patients
}

\author{
Guangshao Cao* \\ Yuyan Liu* \\ Lupeng $\mathrm{Li}$ \\ Xiaoyang Zhao \\ Ruiqing Liu \\ Jian Liu \\ Jianwen Liu \\ Huicun Cao
}

Department of Intervention, Henan Provincial People's Hospital, People's Hospital of Zhengzhou University, People's Hospital of Henan University, Zhengzhou, Henan, People's Republic of China

*These authors contributed equally to this work
Background: There has been very limited investigation regarding the comparison of adverse events (AEs) among radiofrequency ablation (RFA), conventional transarterial chemoembolization (cTACE), and drug-eluting bead TACE (DEB-TACE) in treating HCC patients; therefore, the present study aimed to resolve this issue.

Methods: Two-hundred and forty-six HCC patients (with a total of 267 procedures [treatment times]) treated with RFA (73 patients with 79 procedures), cTACE (86 patients with 94 procedures), or DEB-TACE (87 patients with 94 procedures) were included. Demographic and clinical data were collected. The information on AEs was also retrieved and analyzed.

Results: Total AEs incidence was notably different among the RFA group, cTACE group, and DEB-TACE group and was the highest in cTACE group (86.2\%), then in DEB-TACE group (76.6\%), and the lowest in RFA group (63.3\%). Regarding specific AEs incidence, the incidences of fever, fatigue, and nausea were distinctive among the three groups, while no distinctiveness was found in incidence of other AEs. Furthermore, multivariate logistic regression revealed that cTACE (versus RFA) was independently correlated with increased risk of total AEs, fatigue, and nausea/vomiting; however, the interventional therapies were not independently correlated with the risk of pain, fever or constipation. Other independent predictive factors for total AEs risk were male gender, bronchial asthma, and disease duration.

Conclusion: cTACE resulted in the highest AEs incidence compared with RFA and DEBTACE in treating HCC patients.

Keywords: hepatocellular carcinoma, conventional transarterial chemoembolization, drugeluting bead transarterial chemoembolization, radiofrequency ablation, adverse events

\section{Introduction}

Correspondence: Huicun Cao; Jianwen Liu

Department of Intervention, Henan Provincial People's Hospital, People's Hospital of Zhengzhou University, People's Hospital of Henan University, 7 Weiwu Road, Jinshui District, Zhengzhou, 450003, People's Republic of China

Tel +86-37I-87I602I5

Email caodao0223I5@I63.com;

jian06I409322@I63.com
Hepatocellular carcinoma (HCC) incidence displays a declining trend in some high-risk areas in the world, such as China, while, this phenomenon does not block the way of HCC becoming one of the predominant culprits of cancer-related mortality worldwide. ${ }^{1,2}$ For the purpose of eliminating HCC-related mortality, screening has largely progressed owing to the fact that multiple randomized clinical trials have demonstrated that patients who had experienced a screening program had more favorable survival profiles compared to those who had not. ${ }^{3-5}$ 
However, for individuals with higher risk, there is still a lack of sufficient data suggesting the application of screening, for instance the patients with non-alcoholic fatty liver disease. ${ }^{6,7}$ With respect to the treatment, like in some other solid tumors, HCC patients are treated with surgical resection, palliative therapy, and interventional therapy, the last includes a series of treatments, such as radiofrequency ablation (RFA) and transarterial chemoembolization (TACE), which have experienced great progression in the last few decades. ${ }^{8}$ In addition, some HCC patients could also benefit from hormone replacement therapy. ${ }^{9}$ In order to prolong HCC patients' survival, more effort is applied in improving the treatment modalities technique and promoting advancement in treatment efficacy, however work to decrease unnecessary adverse events is relatively inadequate. Therefore, the tolerance of therapies for HCC patients still needs to be improved.

TACE is a recommended therapy for HCC patients at intermediate stage. ${ }^{8}$ Until now, various types of TACE treatment have been developed, however, only the conventional TACE and drug-eluting bead (DEB)-TACE are frequently applied in clinical practice due to satisfying treatment efficacy and acceptable AEs. ${ }^{10,11}$ As a potential curative therapy, different to TACE, RFA is usually regarded as a therapy for early stage HCC patients who are not fit for hepatic resection. ${ }^{12,13}$ However, RFA is not restricted to this group of patients, it could also be applied in other groups of patients, such as the patients with solitary and deeply seated tumor who should receive RFA to avoid unnecessary resection of the normal liver tissue. $^{14,15}$ In practice, these three therapies are the most applied interventional therapies, which are categorized as minimally invasive procedures. Respectively, their efficacy has been validated in the target groups by multiple studies, including high-quality clinical trials. ${ }^{16-18}$ However, surprisingly, although they all present with acceptable safety profile in the target groups of HCC patients, efforts to compare the safety among RFA, cTACE, and DEB-TACE in HCC patients have been extremely limited.

Hence, in this study, the purpose was to compare the difference of AEs incidence induced by RFA, cTACE, and DEB-TACE, as well as AEs risk factors in HCC patients.

\section{Materials and Methods}

\section{Patients}

This study retrospectively analyzed 246 HCC patients (267 treatment times) who were treated by RFA,
cTACE, or DEB-TACE in our hospital from October 2017 to June 2019. All analyzed patients met the following screening criteria: 1) confirmed diagnosis of HCC according to pathological examination; 2) age $\geq 18$ years old; 3 ) underwent RFA, cTACE, or DEBTACE therapy in our hospital; 4) clinical features data, treatment data, and AEs data available; 5) no other malignant diseases or solid tumors. This study was approved by Institutional Review Board of Henan Provincial People's Hospital (2018-S316), and all patients or their family members provided written informed consent. For those patients who provided informed consent via their family members, it was due to the fact that they were not able to sign the consent themselves, due to various reasons, such as death. In addition, the study was conducted according to the Declaration of Helsinki.

\section{Data Collection}

Medical records of patients were reviewed, and the following data were abstracted: 1) treatment type: RFA, cTACE, and DEB-TACE; 2) demographics: age and gender; 3) underlying disease: hypertension, diabetes mellitus, heart disease, and bronchial asthma; 4) history of HCC: disease duration, surgical history, ablation history, TACE history, and radioactive seeds implantation history; 5) tumor features: tumor size, tumor number and blood supply of tumor; 6) laboratory indexes: total bilirubin (TBIL), alanine transaminase (ALT), aspartate aminotransferase (AST) and creatinine $(\mathrm{Cr})$; 7) treatment information: embolization/ablation status, and total treatment times; 8) AEs: pain, fever, nausea, vomiting, constipation, fatigue, infection, liver abscess, and tumor rupture. According to the common adverse events of RFA, DEB-TACE or cTACE reported in previous studies, any adverse events that occurred during or after the procedures were documented in detail.

\section{Statistical Analysis}

SPSS 22.0 statistical software (IBM, Chicago, Illinois, USA) was applied for data processing and statistical analysis, and GraphPad Prism 7.02 (GraphPad Software Inc., San Diego, California, USA) was used for graphs plotting. Quantitative data were described as mean with standard deviation (SD) or median with interquartile range (IQR) according to the distribution features, meanwhile the qualitative data were described as number as well as percentage (No. (\%)). In the analysis, since some patients 
received repeated treatments, the incidence of AEs in each group was analyzed according to the number of total treatment times. Among the 267 treatment times, the total treatment times of RFA, cTACE, and DEB-TACE were 79, 94, and 94, respectively. Comparison of AEs incidence among three groups was determined by Chisquared test or Fisher's exact test with Bonferroni correction for three-pairwise comparisons. Multivariate logistic regression model analysis was applied to eliminate the effect of confounding factors on the AEs risk. $P$ value $<0.05$ was considered as statistically significant.

\section{Results}

\section{Characteristics of HCC Patients}

The proportions of age $(P=0.134)$ and gender $(P=0.594)$ were similar among the RFA group, cTACE group, and DEB-TACE group (Table 1). In detail, the mean ages were $57.7 \pm 9.5$ years, $58.1 \pm 9.2$ years, and $5.4 \pm 10.1$ years in RFA group, cTACE, and DEB-TACE group, respectively. The numbers of females and males were $16(21.9 \%)$ and 57 (78.1\%), $20(23.3 \%)$ and 66 (76.7\%), 15 (17.2\%) and 72 $(82.8 \%)$ in the three groups, respectively. However, a difference was observed in the aspects regarding disease duration $(P<0.001)$, ablation history $(P<0.001)$, TACE history $(P<0.001)$, radioactive seeds implantation history $(P=0.007)$, tumor size $(P<0.001)$, blood supply of tumor $(P<0.001)$, TBIL level $(P=0.044)$, ALT level $(P=$ $0.001)$, and AST level $(P=0.001)$ among the three groups. Detailed information about the other characteristics of HCC patients was presented in Table 1.

\section{Comparison of AEs Incidence}

Based on the treatment times, incidence of total AEs was notably different among the RFA group, cTACE group, and DEB-TACE group, which were $63.3 \%, 86.2 \%$, and $76.6 \%$, respectively $(P=0.002)$ (Figure 1$)$. Specifically, it was the highest in cTACE group, followed by DEB-TACE group, and was the lowest in RFA group. After adjustment in each two groups, the results displayed that total AEs incidence was elevated in cTACE group compared with RFA group $(P<0.001)$, while, there was no difference between RFA group and DEB-TACE group $(P=0.168)$, nor between cTACE and DEB-TACE group $(P=0.276)$. In terms of specific AEs incidence, the incidence of fever $(P=0.002)$, fatigue $(P<0.001)$, and nausea $(P<0.001)$ was distinguished among the three groups (Table 2). After the adjustment in each two groups, fever $(P=0.027)$, fatigue $(P=0.003)$, and nausea $(P<0.001)$ incidences were all elevated in cTACE group compared to RFA group, meanwhile fever incidence $(P=0.003)$ was higher in DEB-TACE group compared to RFA group, and fatigue incidence $(P<0.001)$ was increased in cTACE group compared to DEB-TACE group.

\section{Comparison of AEs Incidence by Subgroup Analyses}

Based on treatment times, subgroup analyses for assessing the difference of total AEs incidence among the RFA, cTACE, DEB-TACE groups were conducted and showed that the total AEs incidence was different among the three groups in patients with age $\geq 60$ years $(P=0.020)$, male patients $(P=0.002)$, patients without hypertension $(P=$ $0.002)$, patients without diabetes mellitus $(P=0.001)$, patients without heart disease $(P=0.001)$, patients without bronchial asthma $(P=0.01)$, patients with disease duration $\geq 4.0$ months $(P=0.020)$, patients without surgical history $(P=0.010)$, patients without ablation history $(P=0.032)$, patients without TACE history $(P=0.002)$, patients without radioactive seeds implantation history $(P=0.002)$, patients with tumor size $<5.0 \mathrm{~cm}(P=0.013)$, patients with unifocal tumor $(P=0.006)$, patients with moderate blood supply of tumor $(P=0.005)$, patients with abnormal TBIL level $(P=0.034)$, patients with normal ALT level $(P=0.003)$, patients with normal AST level $(P=0.003)$, patients with normal $\mathrm{Cr}$ level $(P=0.007)$, and patients with complete embolism/ablation $(P=0.007)$ (Table 3$)$.

\section{Independent Risk Factors for Total AEs Incidence}

Multivariate logistic regression analysis was conducted based on the treatment times, and it revealed that cTACE (versus RFA) was an independent risk factor for increased total AEs incidence in HCC patients $(P=0.006)$ (Table 4$)$. In addition, male gender $(P=0.032)$, bronchial asthma $(P=0.048)$, and disease duration $\geq 4$ months $(P=0.007)$ could independently predict reduced total AEs incidence.

\section{Independent Risk Factors for Specific AEs Incidence}

Moreover, based on treatment times, independent predictive factors for each specific AE were also evaluated, and the multivariate logistic regression analysis disclosed that, in terms of pain, ablation history $(P=0.002)$ independently predicted decreased risk, however TACE history 
Table I Characteristics of HCC Patients

\begin{tabular}{|c|c|c|c|c|}
\hline Items & RFA (N=73) & cTACE $(\mathrm{N}=86)$ & DEB-TACE $(\mathbf{N}=\mathbf{8 7})$ & $P$ value \\
\hline \multicolumn{5}{|l|}{ Demographics } \\
\hline Age (years), mean $\pm S D$ & $57.7 \pm 9.5$ & $58.1 \pm 9.2$ & $55.4 \pm 10.1$ & 0.134 \\
\hline Gender, No. (\%) & & & & 0.594 \\
\hline Female & $16(21.9)$ & $20(23.3)$ & $15(17.2)$ & \\
\hline Male & $57(78.1)$ & $66(76.7)$ & $72(82.8)$ & \\
\hline \multicolumn{5}{|l|}{ Underlying disease } \\
\hline Hypertension, No. (\%) & $24(32.9)$ & $17(19.8)$ & $20(23.0)$ & 0.144 \\
\hline Diabetes mellitus, No. (\%) & $6(8.2)$ & $10(11.6)$ & $8(9.2)$ & 0.752 \\
\hline Heart disease, No. (\%) & $5(6.8)$ & $5(5.8)$ & $8(9.2)$ & 0.683 \\
\hline Bronchial asthma, No. (\%) & I (I.4) & $\mathrm{I}(\mathrm{I} .2)$ & $2(2.3)$ & 0.822 \\
\hline \multicolumn{5}{|l|}{ History of HCC } \\
\hline Disease duration (months), median (IQR) & $6.0(3.0-24.0)$ & $4.8(2.0-19.3)$ & $2.0(1.0-6.0)$ & $<0.001$ \\
\hline Surgical history, No. (\%) & $22(30.1)$ & $14(16.3)$ & $23(26.4)$ & 0.100 \\
\hline Ablation history, No. (\%) & $34(46.6)$ & $14(16.3)$ & $10(11.5)$ & $<0.001$ \\
\hline TACE history, No. (\%) & $49(67.1)$ & $46(53.5)$ & $28(32.2)$ & $<0.001$ \\
\hline Radioactive seeds implantation history, No. (\%) & $19(26.0)$ & $19(22.1)$ & $7(8.0)$ & 0.007 \\
\hline \multicolumn{5}{|l|}{ Tumor features } \\
\hline Tumor size $(\mathrm{cm})$, mean $\pm \mathrm{SD}$ & $3.7 \pm 2.6$ & $4.8 \pm 3.2$ & $6.5 \pm 3.7$ & $<0.001$ \\
\hline Tumor number, No. (\%) & & & & 0.481 \\
\hline Unifocal & $30(4 I .1)$ & $30(34.9)$ & $38(43.7)$ & \\
\hline Multifocal & $43(58.9)$ & $56(65.1)$ & $49(56.3)$ & \\
\hline Blood supply of tumor, No. (\%) & & & & $<0.001$ \\
\hline Little & $7(9.6)$ & $2(2.3)$ & $2(2.3)$ & \\
\hline Moderate & $57(78.1)$ & $60(69.8)$ & $43(49.4)$ & \\
\hline Large & $9(12.3)$ & $24(27.9)$ & $42(48.3)$ & \\
\hline \multicolumn{5}{|l|}{ Laboratory indexes } \\
\hline TBIL $(\mu \mathrm{mol} / \mathrm{L})$, median $(\mathrm{IQR})$ & $15.0(\mid 1.4-22.5)$ & $16.0(11.5-23.8)$ & $12.7(9.0-20.4)$ & 0.044 \\
\hline ALT (U/L), median (IQR) & $23.0(|7.0-4| .0)$ & $28.0(20.0-38.0)$ & $38.0(23.0-57.0)$ & 0.001 \\
\hline AST (U/L), median (IQR) & $30.0(24.0-45.5)$ & $38.0(29.0-59.0)$ & $43.0(29.0-64.0)$ & 0.001 \\
\hline $\mathrm{Cr}(\mu \mathrm{mol} / \mathrm{L})$, median $(\mathrm{IQR})$ & $56.0(49.0-63.5)$ & $60.0(49.0-68.0)$ & $57.0(49.0-68.0)$ & 0.719 \\
\hline \multicolumn{5}{|l|}{ Treatment information } \\
\hline Complete embolism/ablation, No. (\%) & & & & 0.834 \\
\hline No & $7(9.6)$ & $6(7.0)$ & $7(8.0)$ & \\
\hline Yes & $66(90.4)$ & $80(93.0)$ & $80(92.0)$ & \\
\hline Total treatment times, No. (\%) & 79 & 94 & 94 & 0.951 \\
\hline 1 & $67(91.8)$ & $78(90.7)$ & $80(92.0)$ & \\
\hline 2 & $6(8.2)$ & $8(9.3)$ & $7(8.0)$ & \\
\hline
\end{tabular}

Notes: Comparison was determined by one-way analysis of variance (ANOVA), Chi-squared test or Kruskal-Wallis $H$-test.

Abbreviations: HCC, hepatocellular carcinoma; RFA, radiofrequency ablation; cTACE, conventional transarterial chemoembolization; DEB-TACE, drug-eluting bead transarterial arterial chemoembolization; SD, standard deviation; IQR, interquartile range; TACE, transarterial chemoembolization; TBIL, total bilirubin; ALT, alanine transaminase; AST, aspartate aminotransferase; $\mathrm{Cr}$, creatinine.

$(P=0.046)$ independently predicted increased risk of pain in HCC patients (Supplementary Table 1). As for fever, male gender $(P=0.036)$ independently predicted lower risk but disease duration $\geq 4$ months $(P=0.007)$ independently predicted higher risk of fever (Supplementary Table 2). In regard to fatigue, cTACE (versus RFA) was an independent predictive factor for its increased risk
$(P=0.001)$ (Supplementary Table 3). In addition, heart disease $(P=0.032)$ and complete embolization/ablation $(P=0.020)$ were also independent predictive factors for increased fatigue risk, however, larger blood supply of tumor $(P<0.001)$ was independently correlated with lower risk. As to nausea/vomiting, cTACE $(P=0.001)$ was independently correlated with the increased risk, and 


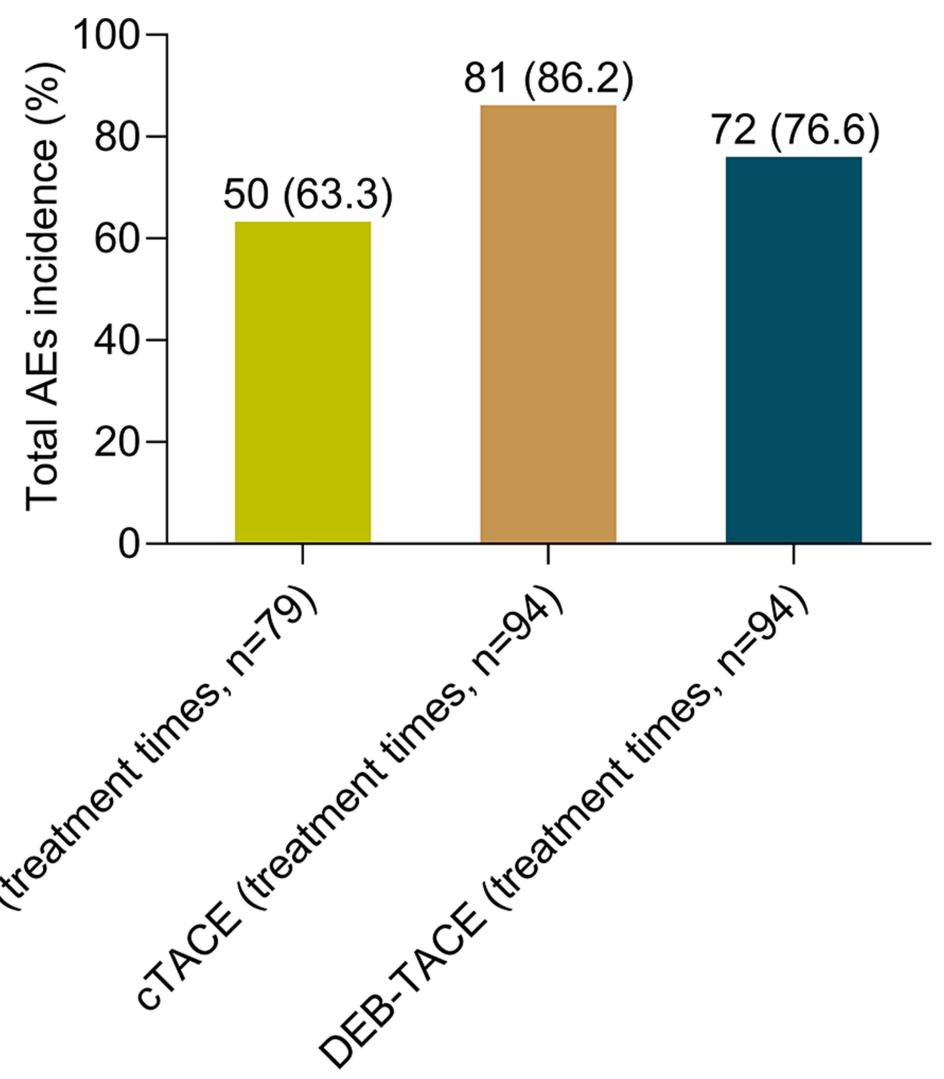

Total: $P=0.002$

RFA vs.cTACE: $P<0.001$

RFA vs.DEB-TACE: $P=0.168$

CTACE vs.DEB-TACE: $P=0.276$

Figure I Total AEs incidence comparison among RFA, cTACE, and DEB-TACE groups. The total AEs incidence was markedly distinctive among the RFA, cTACE, and DEBTACE groups.

Abbreviations: AEs, adverse events; RFA, radiofrequency ablation; cTACE, conventional transarterial chemoembolization; DEB-TACE, drug-eluting bead transarterial chemoembolization.

radioactive seeds implantation history $(P=0.014)$ as well as abnormal ALT level $(P=0.013)$ were also independently correlated with the increased risk (Supplementary Table 4). But abnormal TBIL level $(P=0.033)$ was correlated with decreased risk of nausea/vomiting independently. Moreover, for the risk of constipation, diabetes mellitus $(P=0.003)$ was revealed as an independent predictive factor for the increased risk (Supplementary Table 5).

Table 2 Comparison of Specific AEs Incidence Among Three Groups

\begin{tabular}{|c|c|c|c|c|c|c|c|}
\hline Items & 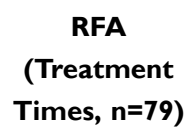 & $\begin{array}{c}\text { cTACE } \\
\text { (Treatment } \\
\text { Times, } n=94 \text { ) }\end{array}$ & $\begin{array}{c}\text { DEB-TACE } \\
\text { (Treatment } \\
\text { Times, n=94) }\end{array}$ & $P$ value* & $P_{\text {value }}^{\#}$ & $P$ value $^{\dagger}$ & P value \& \\
\hline Pain & $30(38.0)$ & $49(52.1)$ & $48(5 I .1)$ & 0.125 & 0.189 & 0.255 & 0.999 \\
\hline Fever, No. (\%) & $18(22.8)$ & $39(4 I .5)$ & 45 (47.9) & 0.002 & 0.027 & 0.003 & 0.999 \\
\hline Fatigue, No. (\%) & $22(27.8)$ & $49(52.1)$ & $23(24.5)$ & $<0.001$ & 0.003 & 0.999 & $<0.001$ \\
\hline Nausea, No. (\%) & $10(12.7)$ & $36(38.3)$ & $21(22.3)$ & $<0.001$ & $<0.001$ & 0.294 & 0.051 \\
\hline Constipation, No. (\%) & $5(6.3)$ & $12(12.8)$ & $5(5.3)$ & 0.136 & 0.471 & 1.000 & 0.225 \\
\hline Vomiting, No. (\%) & $4(5.1)$ & $9(9.6)$ & $5(5.3)$ & 0.395 & 0.786 & 1.000 & 0.798 \\
\hline Liver abscess, No. (\%) & $0(0.0)$ & I (I.I) & $2(2.1)$ & 0.416 & 1.000 & 0.999 & 1.000 \\
\hline Infection, No. (\%) & $0(0.0)$ & $0(0.0)$ & $2(2.1)$ & 0.157 & - & 0.999 & 0.999 \\
\hline Tumor rupture, No. (\%) & $0(0.0)$ & $0(0.0)$ & $\mathrm{I}(\mathrm{I} . \mathrm{I})$ & 0.397 & - & 1.000 & 1.000 \\
\hline
\end{tabular}

Notes: *Comparison among three groups. ${ }^{\#}$ Comparison between RFA group and cTACE group. †Comparison between RFA group and DEB-TACE group. ${ }^{8}$ Comparison between cTACE group and DEB-TACE group. Comparison was determined by Chi-squared test or Fisher's exact test corrected by Bonferroni method.

Abbreviations: AEs, adverse events; RFA, radiofrequency ablation; cTACE, conventional transarterial chemoembolization; DEB-TACE, drug-eluting bead transarterial arterial chemoembolization. 


\begin{tabular}{|c|c|}
\hline 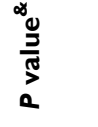 & 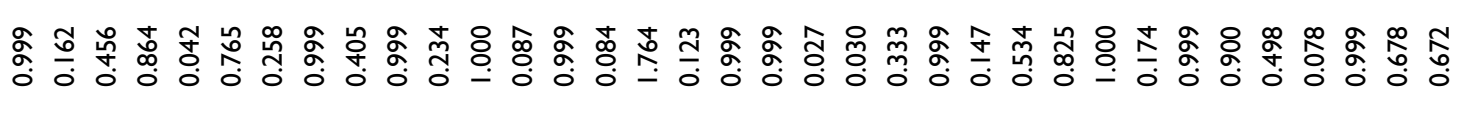 \\
\hline 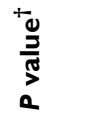 & 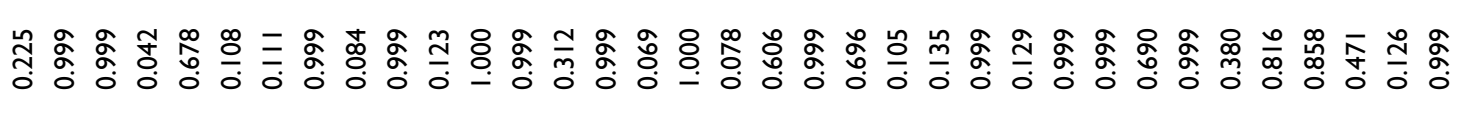 \\
\hline 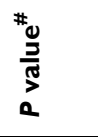 & 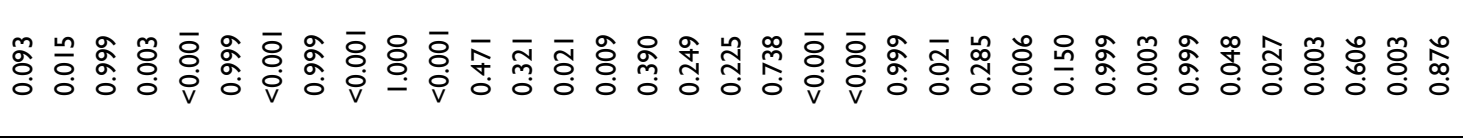 \\
\hline 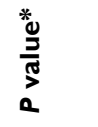 & 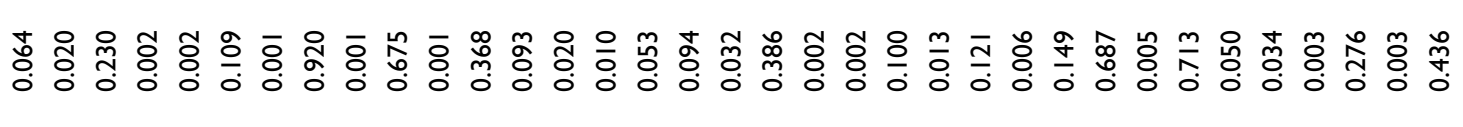 \\
\hline 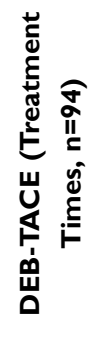 & 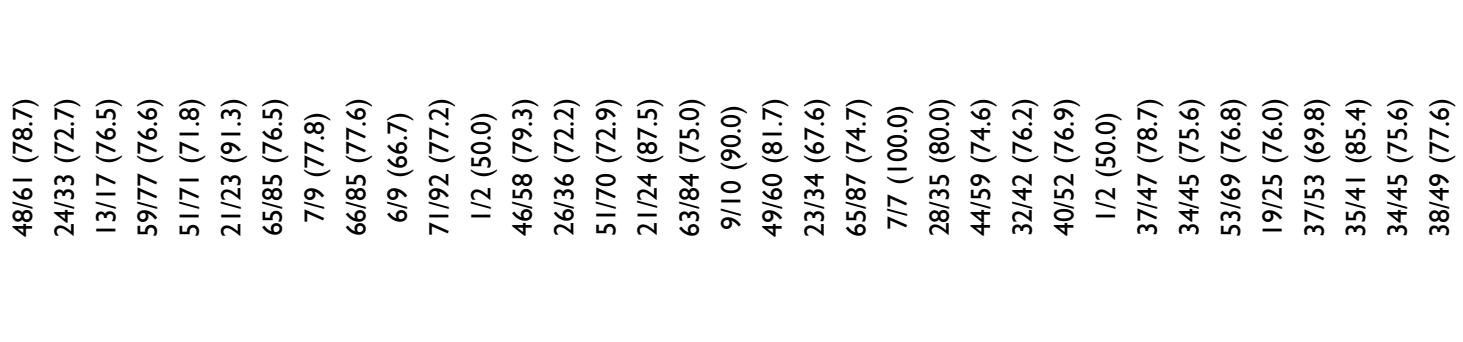 \\
\hline 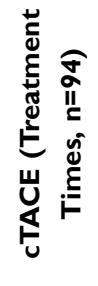 & 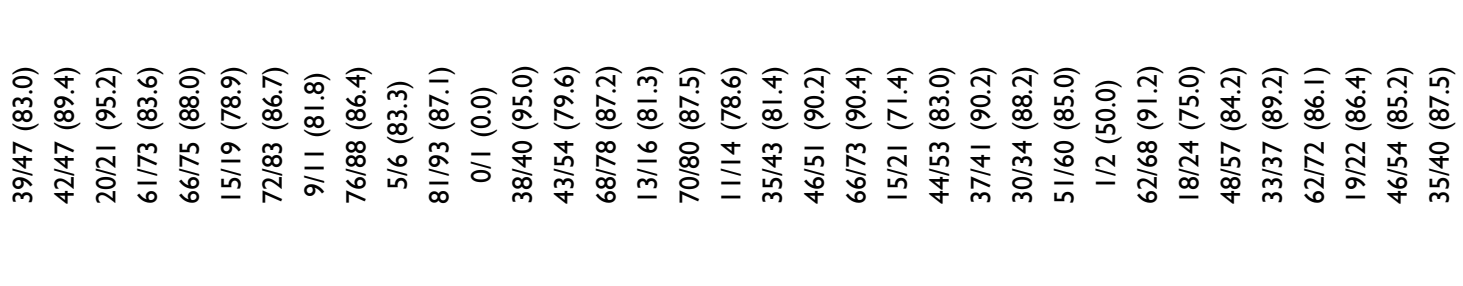 \\
\hline 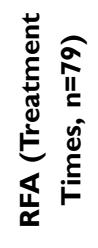 & 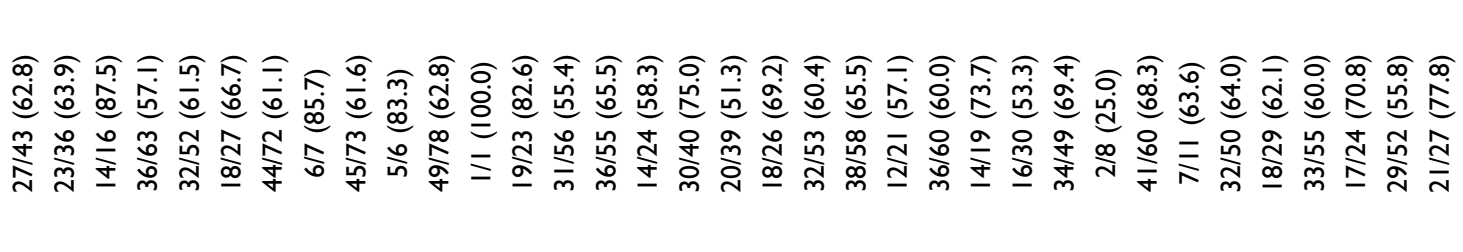 \\
\hline 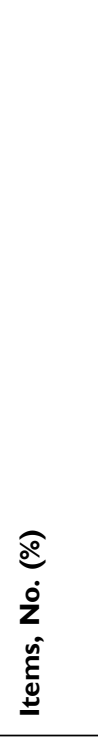 & 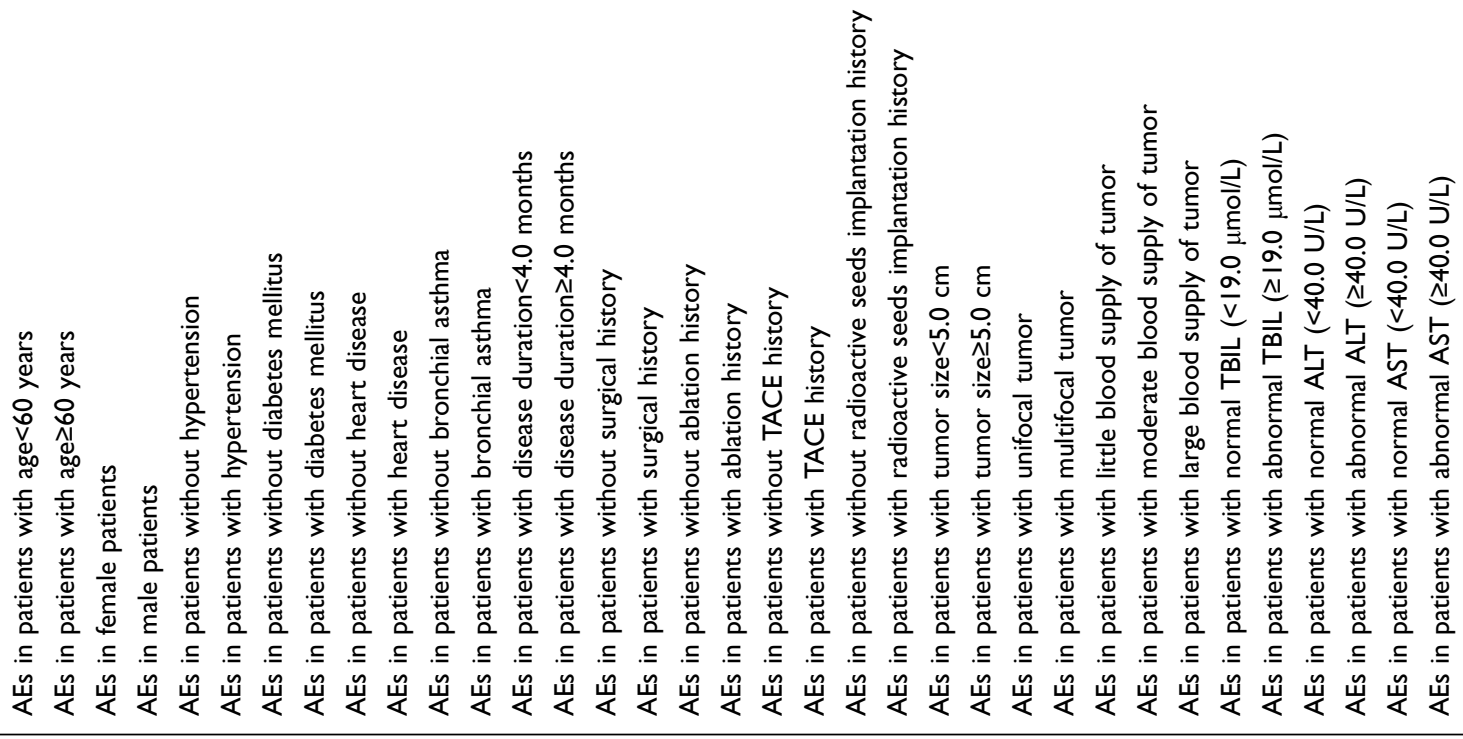 \\
\hline
\end{tabular}




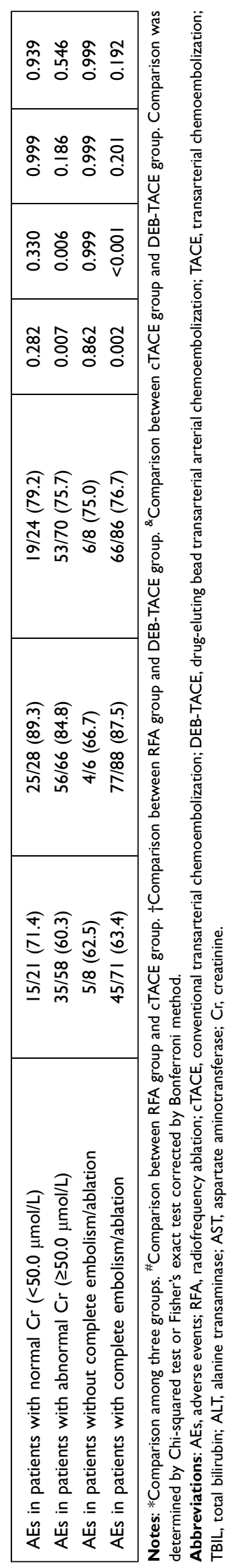

\section{Discussion}

RFA and TACE treatments are therapies generally used in HCC clinical practice thanks to their satisfying efficacy in target patient group, and also the adequate tolerability that ensures the long-term use of these therapies. Additionally, being different from RFA, TACE is a palliative therapy that is usually used in HCC patients who are in the middle stage, therefore most of the studies investigating TACE are focused on middle stage patients. ${ }^{14,19}$ For instance, a recent multicenter cohort study revealed a complication rate of only $2.92 \%$ (330 cases in totally 11,298 procedures) in a large cohort of 9,411 HCC patients treated with RFA. ${ }^{20}$ And for cTACE, a previous retrospective cohort study illuminated that in infiltrative HCC patients treated with cTACE, majority of the AEs were mild (clinical grade 1-2), and the AEs at advanced clinical grade merely occurred in a small proportion of patients, such as hepatic bleeding $(6.1 \%)$ and GI bleeding $(3.0 \%))^{21}$ As for DEB-TACE in HCC patients, a retrospective cohort study reported that in HCC patients who received DEB-TACE, the liver function assessed by laboratory indexes deteriorated in a transient period of less than 3 months, and majority of the AEs were considered as embolization syndrome, which is manageable after supportive care. ${ }^{22}$

To the best of our knowledge, no study has been performed to assess the difference in AEs among RFA, cTACE, and DEB-TACE in HCC patients, however, there are mounting studies that have compared two modalities. For instance, many studies state that DEB-TACE is noninferior to cTACE regarding safety. Additionally, for example, an early randomized controlled study illustrated that the liver toxicity evaluated by laboratory indexes showed no difference between HCC patients treated with DEB-TACE and HCC patients treated with cTACE. $^{23}$ In addition, a retrospective cohort study reported that in HCC patients in 0/A BCLC stage, the clinical grade of abdominal pain was greatly reduced in patients treated with DEB-TACE compared with patients treated with cTACE. ${ }^{24}$ In terms of the safety of RFA versus cTACE or DEB-TACE, a prior retrospective cohort study reported that HCC patients treated with laparoscopic RFA presented with notably decreased incidence of complications compared to patients treated with DEBTACE. ${ }^{25}$ Unfortunately, the studies predominantly aimed at comparing the safety of RFA versus cTACE or DEBTACE are very rare, thus, there are no adequate previous 
Table 4 Multivariate Logistic Regression Analysis of Total AEs Risk

\begin{tabular}{|c|c|c|c|c|}
\hline \multirow[t]{3}{*}{ Items } & \multicolumn{4}{|c|}{ Multivariate Logistic Regression Model } \\
\hline & \multirow[t]{2}{*}{$P$ value } & \multirow[t]{2}{*}{ OR } & \multicolumn{2}{|c|}{$95 \% \mathrm{Cl}$} \\
\hline & & & Lower & Higher \\
\hline \multicolumn{5}{|l|}{ Interventional therapies } \\
\hline RFA & Reference & - & - & - \\
\hline cTACE & 0.006 & 3.291 & $\mathrm{I} .40 \mathrm{I}$ & 7.728 \\
\hline DEB-TACE & 0.586 & 1.268 & 0.540 & 2.978 \\
\hline Age $\geq 60$ years & 0.762 & 0.903 & 0.467 & 1.745 \\
\hline Male gender & 0.032 & 0.331 & 0.121 & 0.907 \\
\hline Hypertension & 0.324 & 1.460 & 0.688 & 3.100 \\
\hline Diabetes mellitus & 0.648 & 1.311 & 0.410 & 4.194 \\
\hline Heart disease & 0.743 & 1.239 & 0.345 & 4.443 \\
\hline Bronchial asthma & 0.048 & 0.098 & 0.010 & 0.980 \\
\hline Disease duration $\geq 4$ months & 0.007 & 0.345 & 0.159 & 0.748 \\
\hline Surgical history & 0.298 & 1.497 & 0.700 & 3.205 \\
\hline Ablation history & 0.250 & 0.621 & 0.276 & 1.398 \\
\hline TACE history & 0.525 & 1.248 & 0.631 & 2.468 \\
\hline Radioactive seeds implantation history & 0.555 & 0.785 & 0.352 & 1.752 \\
\hline Tumor size $\geq 5 \mathrm{~cm}$ & 0.891 & 0.952 & 0.474 & 1.912 \\
\hline Multifocal tumor & 0.166 & 1.593 & 0.825 & 3.078 \\
\hline Larger blood supply of tumor & 0.994 & 0.998 & 0.530 & 1.879 \\
\hline Abnormal TBIL ( $\geq 19.0 \mu \mathrm{mol} / \mathrm{L})$ & 0.687 & 0.869 & 0.438 & 1.722 \\
\hline Abnormal ALT ( $\geq 40.0 \mathrm{U} / \mathrm{L})$ & 0.164 & 1.861 & 0.776 & 4.463 \\
\hline Abnormal AST ( $\geq 40.0$ U/L) & 0.946 & 0.972 & 0.433 & 2.186 \\
\hline Abnormal $\mathrm{Cr}(\geq 50.0 \mu \mathrm{mol} / \mathrm{L})$ & 0.439 & 0.727 & 0.325 & 1.628 \\
\hline Complete embolism/ablation & 0.339 & 1.737 & 0.560 & 5.384 \\
\hline
\end{tabular}

Abbreviations: AEs, adverse events; OR, odds ratio; Cl, confidence interval; RFA, radiofrequency ablation; cTACE, conventional transarterial chemoembolization; DEBTACE, drug-eluting bead transarterial arterial chemoembolization; TACE, transarterial chemoembolization; TBIL, total bilirubin; ALT, alanine transaminase; AST, aspartate aminotransferase; $\mathrm{Cr}$, creatinine.

findings to refer to. Regarding the results in our study, we compared the incidence of total AEs and specific AEs among patients treated with RFA, cTACE, and DEBTACE, then found that the total AEs incidence presented with notable difference among RFA, cTACE, DEB-TACE groups, and was the highest in cTACE group. This suggested that cTACE might be the least tolerable treatment in HCC patients when compared with RFA and DEB-TACE. In regard to probable explanations, we speculated that the result of total AEs incidence being the highest in cTACE group may be related to the technical process of cTACE, including the application of lipiodol and large size embolization particles, which could both contribute to a higher AEs incidence. ${ }^{21,26}$ Furthermore, we also found a difference in incidence of fever, fatigue, and nausea among the three therapies, and to be specific, the incidences of fever, fatigue, and nausea were increased in cTACE group compared to RFA group, fever incidence was increased in DEB-TACE group in comparison to RFA group, and fatigue incidence was elevated in cTACE group compared to DEB-TACE group. These findings indicated that RFA was superior to cTACE and DEB-TACE in HCC patients regarding fever, fatigue, and nausea incidences, which could provide some information when it comes to decision-making in the management of HCC patients. However, these differences in fever, fatigue, and nausea incidences in RFA, cTACE, and DBE-TACE treatment in HCC patients should be validated by clinical trials and cohort studies with larger sample size.

Another aspect worth exploring is whether the characteristics of HCC patients have an influence on the AEs incidence in RFA, cTACE, and DEB-TACE treatments. Therefore, the subgroup analysis was performed, which disclosed that total AEs incidence was distinctive in patients with different demographic characteristics (age and gender), clinical features (complication types, disease duration, treatment history, and tumor features), liver function related laboratory indexes levels (ALT and AST 
levels), renal function related laboratory index level $(\mathrm{Cr}$ level), and situation of complete embolization/ablation. In addition, male gender was independently correlated with increased fever risk in this study. Although we still have insufficient clues regarding the correlation between male gender and fever, this finding still provides information on the prevention of fever in HCC patients in practice, besides, there have been studies that revealed that male gender is correlated with increased AEs risk in HCC patients treated with TACE. ${ }^{19}$ These data signify that these features of HCC patients should be included in the consideration of treatment modality selection when it involves RFA, cTACE, and DEB-TACE. However, although this is valuable information for clinical practice, it still needs further establishment by more clinical studies. In addition, in regard to the management of AEs, the most common AEs, such as embolization syndrome, is relatively easy to manage; however, several of the other AEs, such as fatigue, is relatively troublesome because there is still no drug that can efficiently cure fatigue.

Moreover, we also observed that cTACE was independently correlated with increased risk of total AEs, fatigue, and nausea/vomiting, this could result from the features of cTACE treatment involving the use of lipiodol and large size embolization particles, as explained previously. $^{21,26}$ Moreover, we also found that male gender, bronchial asthma, heart disease, diabetes mellitus, disease duration, blood supply of tumor, ablation history, TACE history, radioactive seeds implantation history, TBIL level, ALT level, and complete embolization/ablation were also independent predictive factors for total AEs or specific AEs. These probably could provide more information on the management of HCC patients regarding choosing the optimal therapy to maximize the elimination of AEs. In addition, an issue which should be discussed is that the indication of RFA and TACE was somewhat different. However, from another aspect, RFA, DEB-TACE, and cTACE were all part of the interventional therapy which was carried out in the same Department (Department of Intervention) in China, therefore, it was interesting to find out the safety difference among them, which might provide more evidence about the interventional therapy for HCC and for the reference of Department of Intervention.

There were several limitations in the current study that we would like to discuss. 1) A total of 267 procedures (treatment times) were analyzed in this study, which was a relatively small sample size and may have had inadequate statistical power. 2) Several characteristics of HCC patients varied among the three groups, which might interfere with the results regarding the AEs incidence. However, considering the distinctive target patient groups, it was difficult to enroll patients with matched clinical features or treatment histories. 3) The observational time was relatively short in our study, which might result in an unclear difference in the long-term AEs in HCC patients treated with the three modalities assessed in our study. 4) Since the present study was a retrospective cohort study, recall bias from the patients and documentation bias from the clinicians or nurses responsible for recording the patients' information might exist. 5) Besides, comparing the AEs in RFA and TACE treatments might be a limitation as well, due to the fact that the targeted patient population receiving RFA and TACE treatments is distinctive. 6) Due to the retrospective design, it was not possible to fully retrieve the grade of AEs for grade evaluation using NCI CTCAE criteria, which needs to be explored in future studies.

In conclusion, cTACE resulted in the highest AEs incidence compared with RFA and DEB-TACE in HCC patients. These findings may facilitate the decision-making and AEs prevention in HCC treatment, since we found a difference regarding AEs incidence among the three treatments in HCC patients, and several clinical features were correlated with increased or decreased AEs incidence. Also, future studies should focus on investigating the AEs induced by cTACE compared with RFA and DEB-TACE in a larger cohort.

\section{Acknowledgments}

This study was supported by Henan Provincial Medical Science and Technique Foundation (No.2018020392).

\section{Disclosure}

The authors declare that they have no conflicts of interest.

\section{References}

1. Kulik L, El-Serag HB. Epidemiology and management of hepatocellular carcinoma. Gastroenterology. 2019;156(2):477-491 e471. doi:10.1053/j.gastro.2018.08.065

2. Llovet JM, Zucman-Rossi J, Pikarsky E, et al. Hepatocellular carcinoma. Nat Rev Dis Primers. 2016:216018. doi:10.1038/ $\operatorname{nrdp} .2016 .18$ 
3. Sherman M. Surveillance for hepatocellular carcinoma. Best Pract Res Clin Gastroenterol. 2014;28(5):783-793. doi:10.1016/j. bpg.2014.08.008

4. Croswell JM, Ransohoff DF, Kramer BS. Principles of cancer screening: lessons from history and study design issues. Semin Oncol. 2010;37(3):202-215. doi:10.1053/j.seminoncol.2010.05.006

5. Zhang BH, Yang BH, Tang ZY. Randomized controlled trial of screening for hepatocellular carcinoma. J Cancer Res Clin Oncol. 2004;130(7):417-422. doi:10.1007/s00432-004-0552-0

6. Degasperi E, Colombo M, Doyle A, Gorgen A, Muaddi H. Distinctive features of hepatocellular carcinoma in non-alcoholic fatty liver disease. Lancet Gastroenterol Hepatol. 2018;18 (1):156-164. doi:10.1016/S2468-1253(16)30018-8

7. European Association for the Study of the L, European Association for the Study of D, European Association for the Study of O. EASLEASD-EASO Clinical Practice Guidelines for the management of non-alcoholic fatty liver disease. $J$ Hepatol. 2016;64(6):1388-1402. doi:10.1016/j.jhep.2015.11.004

8. Hsu CY, Liu PH, Ho SY, et al. Using nomogram of the barcelona clinic liver cancer system for treatment selection in patients with stage C hepatocellular carcinoma. BMC Cancer. 2018;18(1):289. doi:10.1186/s12885-018-4202-3

9. Teng TZJ, Shelat VG. Testosterone gel improves appetite and reduces tiredness in males with advanced cancer. BMJ Support Palliat Care. 2021;11(2):145. doi:10.1136/bmjspcare-2020-002662

10. Zhou Y. Drug-eluting bead transarterial chemoembolization is efficient and well-tolerated in treating elderly Chinese hepatocellular carcinoma patients. Int J Clin Exp Pathol. 2018;11(10):4867-4878.

11. Liu YS, Lin CY, Chuang MT, et al. Five-year outcome of conventional and drug-eluting transcatheter arterial chemoembolization in patients with hepatocellular carcinoma. BMC Gastroenterol. 2018;18 (1):124. doi:10.1186/s12876-018-0848-1

12. Doyle A, Gorgen A, Muaddi H, et al. Outcomes of radiofrequency ablation as first-line therapy for hepatocellular carcinoma less than $3 \mathrm{~cm}$ in potentially transplantable patients. J Hepatol. 2019;70 (5):866-873. doi:10.1016/j.jhep.2018.12.027

13. Uhlig J, Sellers CM, Stein SM, Kim HS. Radiofrequency ablation versus surgical resection of hepatocellular carcinoma: contemporary treatment trends and outcomes from the United States national cancer database. Eur Radiol. 2019;29(5):2679-2689. doi:10.1007/s00330-018-5902-4

14. Gui CH, Baey S, D’Cruz RT, Shelat VG. Trans-arterial chemoembolization + radiofrequency ablation versus surgical resection in hepatocellular carcinoma - a meta-analysis. Eur J Surg Oncol. 2020;46 (5):763-771. doi:10.1016/j.ejso.2020.01.004

15. Lei GY, Junnarkar LS, Huey T, Shelat V. Predictors of 90-day mortality following hepatic resection for hepatocellular carcinoma. Visc Med. 2020. doi:10.1159/000510811
16. Zhang L, Ge NL, Chen Y, et al. Long-term outcomes and prognostic analysis of radiofrequency ablation for small hepatocellular carcinoma: 10-year follow-up in Chinese patients. Med Oncol. 2015;32 (3):77. doi:10.1007/s12032-015-0532-z

17. Cosgrove DP, Reyes DK, Pawlik TM, Feng AL, Kamel IR, Geschwind JF. Open-label single-arm phase II trial of sorafenib therapy with drug-eluting bead transarterial chemoembolization in patients with unresectable hepatocellular carcinoma: clinical results. Radiology. 2015;277(2):594-603. doi:10.1148/radiol.2015142481

18. Kloeckner R, Weinmann A, Prinz F, et al. Conventional transarterial chemoembolization versus drug-eluting bead transarterial chemoembolization for the treatment of hepatocellular carcinoma. $B M C$ Cancer. 2015:15465. doi:10.1186/s12885-015-1480-x

19. Ahmed S, de Souza NN, Qiao W, Kasai M, Keem LJ, Shelat VG. Quality of life in hepatocellular carcinoma patients treated with transarterial chemoembolization. HPB Surg. 2016;20166120143. doi:10.1155/2016/6120143

20. Maeda M, Saeki I, Sakaida I, et al. Complications after radiofrequency ablation for hepatocellular carcinoma: a Multicenter Study Involving 9411 Japanese patients. Liver Cancer. 2020;9(1):50-62. doi:10.1159/000502744

21. Zhang ZS, Li HZ, Ma C, Xiao YD. Conventional versus drug-eluting beads chemoembolization for infiltrative hepatocellular carcinoma: a comparison of efficacy and safety. BMC Cancer. 2019;19(1):1162. doi:10.1186/s12885-019-6386-6

22. Chen S, Ji R, Shi X, Wang Z, Zhu D. Retrospective analysis of efficacy, safety, and prognostic factors in a cohort of Chinese hepatocellular carcinoma patients treated with drug-eluting bead transarterial chemoembolization. Braz J Med Biol Res. 2019;52(12):e8467. doi:10.1590/1414-431x20198467

23. Sacco R, Bargellini I, Bertini M, et al. Conventional versus doxorubicin-eluting bead transarterial chemoembolization for hepatocellular carcinoma. J Vasc Interv Radiol. 2011;22(11):1545-1552. doi:10.1016/j.jvir.2011.07.002

24. Kang YJ, Lee BC, Kim JK, et al. Conventional versus small doxorubicin-eluting bead transcatheter arterial chemoembolization for treating barcelona clinic liver cancer stage 0/A hepatocellular carcinoma. Cardiovasc Intervent Radiol. 2020;43(1):55-64. doi:10.1007/s00270-019-02349-9

25. Hsieh CB, Chang HM, Chen TW, et al. Comparison of transcatheter arterial chemoembolization, laparoscopic radiofrequency ablation, and conservative treatment for decompensated cirrhotic patients with hepatocellular carcinoma. World J Gastroenterol. 2004;10 (4):505-508. doi:10.3748/wjg.v10.i4.505

26. Imai N, Ishigami M, Ishizu Y, et al. Transarterial chemoembolization for hepatocellular carcinoma: a review of techniques. World J Hepatol. 2014;6(12):844-850. doi:10.4254/wjh.v6.i12.844

\section{Publish your work in this journal}

Cancer Management and Research is an international, peer-reviewed open access journal focusing on cancer research and the optimal use of preventative and integrated treatment interventions to achieve improved outcomes, enhanced survival and quality of life for the cancer patient.
The manuscript management system is completely online and includes a very quick and fair peer-review system, which is all easy to use. Visit http://www.dovepress.com/testimonials.php to read real quotes from published authors. 